\title{
Correlation between the tuberculin skin test and T-SPOT.TB in patients with suspected tuberculosis infection: A pilot study
}

\author{
JING YANG ${ }^{1}$, WEILIANG KONG ${ }^{1}$, NING XV $^{1}$, XIAOPING HUANG ${ }^{1}$ and XUEQING CHEN ${ }^{2}$ \\ Departments of ${ }^{1}$ Respiratory Medicine and ${ }^{2}$ Traditional Medicine, Ningbo First Hospital, Ningbo, Zhejiang 315010, P.R. China
}

Received December 28, 2018; Accepted June 13, 2019

DOI: $10.3892 /$ etm.2019.7791

\begin{abstract}
T-SPOT.TB is a novel screening method for Mycobacterium tuberculosis infection. However, it is controversial whether T-SPOT.TB should become an alternative method to the tuberculin skin test (TST) for screening M. tuberculosis infections. The present study aimed to evaluate this issue based on the retrospective analysis of clinical cases. TST and T-SPOT.TB tests were used on patients with suspected $M$. tuberculosis infection on admission. Demographic data and clinical information, including previous history of $M$. tuberculosis infection, were collected. A total of 118 patients were included in the analysis, among whom 30 (25.4\%) were diagnosed with active $M$. tuberculosis infection, and seven patients $(5.9 \%)$ were currently receiving immunosuppressive treatment. The overall sensitivity and specificity of the TST were 76.7 and $77.3 \%$, respectively, while they were 88.3 and $68.1 \%$, respectively, for the T-SPOT.TB test. Patients with large TST indurations had a higher number of gamma interferon-producing $\mathrm{T}$ cells among peripheral blood mononuclear cells compared with those of TST-negative patients. In conclusion, the T-SPOT.TB test had a higher sensitivity than the TST, but the difference was not statistically significant. Neither the T-SPOT.TB test nor the TST was sufficiently accurate to detect active M.tuberculosis infection.
\end{abstract}

\section{Introduction}

Mycobacterium tuberculosis (TB) infection remains a large global health problem. In 2017, an estimated 10.0 million individuals developed TB. TB is now the 10th leading cause of death worldwide and the leading cause of mortality from a single infectious agent. China is one of 30 high TB-burden

Correspondence to: Dr Xiaoping Huang, Department of Respiratory Medicine, Ningbo First Hospital, 59 Liuting Street, Ningbo, Zhejiang 315010, P.R. China

E-mail: xiaopinghuang@126.com

Dr Xueqing Chen, Department of Traditional Medicine, Ningbo First Hospital, 59 Liuting Street, Ningbo, Zhejiang 315010, P.R. China E-mail: cxq2316@163.com

Key words: $M$. tuberculosis infection, tuberculosis, interferon- $\gamma$ release assay, T-SPOT.TB, tuberculin skin test countries (1). To reach the goal of TB elimination, individuals with active TB require rapid identification and treatment. Microscopy, growth in culture and molecular tests are the gold standards for the diagnosis of active TB, as they directly indicate the presence of actual TB bacilli or their DNA $(1,2)$. However, not all cases of TB infection may be bacteriologically confirmed. For patients with a negative acid-resistant bacillus sputum-smear test, diagnosis and treatment decisions may be challenging.

The tuberculin skin test (TST) has been widely used for detecting latent TB infection (LTBI) and active TB for almost a century. The important advantages of the TST include its low cost and convenience. However, the TST result may be influenced by prior Bacillus Calmette-Guerin (BCG) vaccination and infection with non-tuberculous mycobacteria (NTM) (3). In recent years, several commercially available interferon- $\gamma$ release assays (IGRAs) have been developed as an alternative screening approach for TB infection. These tests, including the T-SPOT.TB test, QuantiFERON-TB Gold or QuantiFERON-TB Gold In-Tube target unique and specific M.tuberculosis proteins that are not present in BCG or in most environmental mycobacteria (3). Several meta-analyses have indicated a relatively enhanced sensitivity and specificity of IGRAs over the TST in identifying TB infection. However, neither the IGRAs nor the TST exhibited ideal stability (4-6).

To assess the value of these two methods, the present retrospective analysis was performed. The performance of the T-SPOT.TB in detecting active TB was compared with that of the TST and the comparison between these two detection methods was determined.

\section{Materials and methods}

Participants and data collection. A retrospective analysis was performed on patients diagnosed at the Respiratory Department of Ningbo First Hospital (Ningbo, China) between October 2016 and 2017. A total of 118 patients who were suspected of active TB infection on admission were included in the analysis. Each patient was subjected to the TST as well as the T-SPOT.TB test. The patients' demographics and clinical information, including previous history of TB, were collected.

Definitions and diagnoses. Final diagnoses were made considering all clinical,radiological, microbiological and pathological information. Patients who had clinical, bacteriological and/or 
radiographic evidence of active $\mathrm{TB}$ infection were defined as active TB cases of the following varieties: i) Pulmonary TB: M. tuberculosis was cultured from sputum, bronchial specimens or patients whose data met the definition of a clinical case of TB (7). For clinical cases of TB, chest radiographic findings were defined as the presence of cavities, branching linear lesions, multiple centrilobular nodules or lobular consolidation upon high resolution CT $(8,9)$. Lesions that mostly appeared as calcified nodules or fibrotic scars were not considered to be indicative of active TB infection. For patients with lesions that suggested active TB but who had a negative bacteriologic status, broad-spectrum antibiotics were given for one week. If the lesions significantly improved, a diagnosis of active TB was ruled out. All clinical cases were followed up for at least 3 months for further confirmation. ii) Pleural TB: M. tuberculosis was detected in the pleural fluid or a tissue biopsy, or exudative pleural effusion exhibited predominant lymphocytosis, high protein, low carcinoembryonic antigen $(<5 \mathrm{ng} / \mathrm{ml})$ and high adenosine deaminase ( $\geq 40 \mathrm{IU} / \mathrm{l})(7,10)$. iii) Lymph node TB: M. tuberculosis was detected in lymph node tissue.

TST and T-SPOT.TB. A TST was performed following standard procedures. A total of $0.1 \mathrm{ml}$ of purified protein derivate (Chengdu Institute of Biological Products Co., Ltd.) was injected intradermally into the inner side of the forearm, and the transverse induration was measured in $\mathrm{mm}$ after $48-72 \mathrm{~h}$ by trained nurses. An induration of $\geq 10 \mathrm{~mm}$ (or $\geq 5 \mathrm{~mm}$ in immunosuppressed individuals) was classified as a positive result. The T-SPOT.TB assays (Beijing Wan Tai Bio-Pharmaceutical Co., Ltd) were performed and interpreted according to the manufacturer's specifications (11).

Statistical analysis. Continuous variables are expressed as the mean and standard deviation. Frequencies were calculated for demographic and clinical data. Comparisons between different groups were performed using a Student's t-test or least-significant difference (LSD) test. The LSD test was performed following one-way analysis of variance. Fisher's exact or $\chi^{2}$ tests were used for univariate analyses. In each analysis, $\mathrm{P}<0.05$ was considered to indicate a statistically significant difference. Statistical analyses were performed using SPSS for Windows, version 22.0 (IBM Corp.) and GraphPad Prism 5.0 (GraphPad Software, Inc.).

\section{Results}

Characteristics of the study population. A total of 118 patients were included in the analysis and $70(59.3 \%)$ of them were female. The median age was 56.5 years (range, 18-95 years). One patient $(0.8 \%)$ was confirmed as HIV-positive and seven patients $(5.9 \%)$ were currently receiving immunosuppressive treatment. A total of 10 patients $(8.5 \%)$ had previously been diagnosed with TB and had received anti-TB treatment. BCG scars were present in 88 patients $(74.6 \%)$. Active TB infection was diagnosed in 30 patients $(25.4 \%)$ and 15 of them had pulmonary TB. A total of 88 patients $(74.6 \%)$ were diagnosed with non-TB conditions. Pneumonia was the most common disease among those non-TB cases. Concerning the laboratory data, TB patients had a lower lymphocyte ratio than that in the
non-TB group. There were no significant differences between the $\mathrm{CD}^{+}$lymphocyte ratio and the $\mathrm{CD} 8^{+}$lymphocyte ratio between the two groups (Table I).

Performance of T-SPOT.TB and TST in active TB. Of all of the 118 patients, the TST results were positive for 43 patients (36\%), 23 of whom were diagnosed with active TB; the TST results were negative for 75 patients (64\%), 7 of whom were diagnosed with active TB. The overall sensitivity and specificity of the TST were 76.7 and $77.3 \%$, respectively. In the T-SPOT.TB test, 53 patients (45\%) had positive results, 25 of whom were diagnosed with active TB; 65 patients (55\%) had negative results, 5 of whom were diagnosed with active TB. The overall sensitivity and specificity of the T-SPOT.TB test were 88.3 and $68.1 \%$, respectively. However, no significant difference was observed between T-SPOT.TB and TST (Table II).

The accuracy of the TST and T-SPOT.TB test for pulmonary and extrapulmonary TB (EPTB) was calculated separately. For pulmonary TB, the sensitivity of the TST was $80.0 \%$, and the specificity was $70.0 \%$. The sensitivity and specificity of the T-SPOT.TB test was 80.0 and $60.2 \%$, respectively. For the EPTB, the TST sensitivity was $73.3 \%$ and the specificity was $68.9 \%$, while the sensitivity and specificity of the T-SPOT. TB were 86.7 and $61.2 \%$, respectively. No significant difference was noted in the above results between the TST and the T-SPOT.TB test. The negative predictive value (NPVs) of the TST and T-SPOT.TB test was higher than the respective positive predictive value (PPVs) (Table II).

Association between the TST spot size and T-SPOT.TB results. There was a trend toward an increased likelihood of T-SPOT. TB positivity with increased TST spot size. Patients with a large TST size $(>20 \mathrm{~mm})$ had a higher number of gamma interferon-producing $\mathrm{T}$ cells among their peripheral blood mononuclear cells (PBMCs) than that of TST-negative patients (spot size, $<10 \mathrm{~mm} ; 240.2 \pm 155.6$ vs. 101.4 \pm 129.1 ; spot-forming cells $\left./ 10^{6} \mathrm{PBMCs}, \mathrm{P}=0.008\right)$. However, no such differences were observed among other stratified data based on the TST size (Table III and Fig. 1).

\section{Discussion}

The major results of the present study were as follows: i) The T-SPOT.TB test had a higher sensitivity than the TST, but the specificity and PPV were comparatively lower than those of the TST. However, none of the above results were statistically significant; ii) the NPVs of the TST and the T-SPOT.TB test were much higher than the PPVs; and iii) increased TST spot size is associated with a trend toward increased rates of T-SPOT.TB positivity.

The overall sensitivity of the TST and T-SPOT.TB test were 76.7 and $88.3 \%$, respectively, in the present study. The T-SPOT.TB test had a comparatively higher sensitivity than that of the TST, which was consistent with the results of certain previous meta-analyses $(6,12)$. However, compared to most data for cohorts from developed countries $(3,12)$, a lower specificity (68.1\%) and PPV (47.2\%) of the T-SPOT.TB was determined in the present study, which is more consistent with the result of one large-scale retrospective multicenter study from China (13). The accuracy of the TST and T-SPOT. 
Table I. Baseline characteristics of the subjects.

\begin{tabular}{|c|c|c|c|}
\hline Characteristic & $\mathrm{TB}(\mathrm{n}=30)$ & Non-TB $(n=88)$ & P-value \\
\hline Male sex & $14(47.7)$ & $56(63.7)$ & 0.102 \\
\hline Age, years & $50.0 \pm 19.5$ & $58.7 \pm 17.4$ & 0.024 \\
\hline BMI & $20.7 \pm 3.8$ & $21.2 \pm 3.6$ & 0.485 \\
\hline Smoking index & $198.5 \pm 350.3$ & $283.8 \pm 503.8$ & 0.393 \\
\hline Prior treatment of TB & $3(10.0)$ & $7(8.0)$ & 0.728 \\
\hline Current immunosuppressive treatment & $4(13.3)$ & $3(3.4)$ & 0.047 \\
\hline BCG scar present & $18(60.0)$ & $70(79.5)$ & 0.100 \\
\hline WBC $\left(10^{9} / 1\right)$ & $6.7 \pm 1.9$ & $7.3 \pm 4.0$ & 0.404 \\
\hline Lymphocytes (\%) & $19.0 \pm 6.4$ & $23.3 \pm 9.7$ & 0.007 \\
\hline $\mathrm{CRP}(\mathrm{mg} / \mathrm{dl})$ & $20.0 \pm 22.1$ & $27.2 \pm 48.4$ & 0.273 \\
\hline $\mathrm{CD}^{+}{ }^{+} \mathrm{T}$ lymphocytes $(\%)$ & $41.4 \pm 10.5$ & $40.3 \pm 8.8$ & 0.736 \\
\hline $\mathrm{CD}^{+} \mathrm{T}$ lymphocytes $(\%)$ & $24.1 \pm 11.1$ & $24.6 \pm 10.3$ & 0.900 \\
\hline \multicolumn{4}{|l|}{ Final diagnosis } \\
\hline Pulmonary TB & $14(46.7)$ & & \\
\hline Endobronchial TB & $1(3.3)$ & & \\
\hline Pleural TB & $13(43.3)$ & & \\
\hline Lymph node TB & $2(6.7)$ & & \\
\hline Pneumonia & & $65(73.9)$ & \\
\hline Pulmonary fungal infection & & $4(4.5)$ & \\
\hline Sarcoidosis & & $2(2.3)$ & \\
\hline Lung tumor & & $6(6.8)$ & \\
\hline Others & & $11(12.5)$ & \\
\hline
\end{tabular}

Values are expressed as the mean \pm standard deviation or $\mathrm{n}(\%)$. Percentages are calculated for the number in each subgroup within the same column. TB, tuberculosis; TST, tuberculin skin test; WBC, white blood cells; BMI, body mass index; BCG, Bacillus Calmette-Guerin; CRP, C-reactive protein.

Table II. Performance of T-SPOT.TB and TST in active TB.

\begin{tabular}{lrrc}
\hline Parameter (\%) & T-SPOT.TB & TST & P-value \\
\hline Overall sensitivity & $83.3(25 / 30)$ & $76.7(23 / 30)$ & 0.51 \\
Overall specificity & $68.1(60 / 88)$ & $77.3(68 / 88)$ & 0.17 \\
Pulmonary TB & & & \\
Sensitivity & $80.0(12 / 15)$ & $80.0(12 / 15)$ & 1 \\
Specificity & $60.2(62 / 103)$ & $70.0(72 / 103)$ & 0.14 \\
PPV & $23.6(12 / 53)$ & $27.9(12 / 43)$ & 0.55 \\
NPV & $95.4(62 / 65)$ & $96.0(72 / 75)$ & 0.86 \\
Extrapulmonary TB & & & \\
Sensitivity & $86.7(13 / 15)$ & $73.3(11 / 15)$ & 0.41 \\
Specificity & $61.2(63 / 103)$ & $68.9(71 / 103)$ & 0.24 \\
PPV & $24.5(13 / 53)$ & $25.6(11 / 43)$ & 0.91 \\
NPV & $96.9(63 / 65)$ & $94.7(71 / 75)$ & 0.51 \\
\hline
\end{tabular}

TB, tuberculosis; TST, tuberculin skin test; NPV, negative predictive value; PPV, positive predictive value.

TB test for EPTB was also evaluated. The specificity of the T-SPOT.TB test did not exhibit any advantage over that of the TST (61.2 vs. $68.9 \%)$, which may indicate a relatively high prevalence of LTBI in the region of residence of the present cohort. The T-SPOT.TB test and the TST are based on cellular immune responses, and they are unable to distinguish between latent TB infection and active TB (14). Therefore, the T-SPOT. TB may have limited value in detecting active TB, particularly in high TB burden settings $(15,16)$.

It was found that the NPVs were much higher than the PPVs for the TST (91 vs. 53\%) and for the T-SPOT.TB test (92 vs. 47\%). This indicated that the T-SPOT.TB test and the TST may be more appropriate for ruling out active TB than for ruling it in. A previous study suggested that the combination of negative results obtained by IGRAs with the TST may enable the rapid exclusion of TB $(17,18)$. Further studies are required to identify the optimal combined strategy for targeted screening. However, considering the low PPV of the two methods, active TB should not simply be excluded for high-risk individuals without a thorough microbiological examination of M.tuberculosis.

In the present study, a trend toward an increased likelihood of a positive T-SPOT.TB result with increased TST spot size was observed. Several studies have evaluated the association between the TST size and IGRAs result in LTBI, and the results indicated that the TST size may help identify those subjects with the highest risk of LTBI (19-21). All of these studies lack the gold standard testing for determining LTBI. The present study identified a relative concordance of a positive T-SPOT.TB result and the TST size in detecting active TB. Patients with a TST size of 
Table III. Comparison between size of TST induration and T-SPOT.TB result.

T-SPOT.TB

\begin{tabular}{lccc} 
TST induration $(\mathrm{mm})$ & $\mathrm{N}$ & Positive $\mathrm{n}(\%)$ & SFCs/10 ${ }^{6} \mathrm{PBMC}$, mea \\
\hline$<10$ & 75 & $18(24.0)$ & $101.4 \pm 129.1$ \\
$\geq 10$ & 43 & $34(79.1)$ & $196.7 \pm 134.9$ \\
& & & ${ }^{\mathrm{P}}=0.017$ \\
$10-14$ & 22 & $16(72.7)$ & $168.9 \pm 112.0$ \\
$15-19$ & 6 & $5(83.3)$ & $172.8 \pm 143.1$ \\
$\geq 20$ & 15 & $13(86.7)$ & $240.2 \pm 155.6$ \\
& & & bP $=0.297$
\end{tabular}

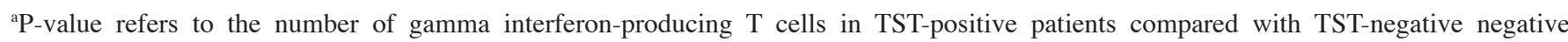
patients. ${ }^{b} \mathrm{P}$-value refers to the differences among stratified data based on TST spot size. TST, tuberculin skin test; SFCs, spot-forming-cells; PBMC, peripheral blood mononuclear cells; $\mathrm{SD}$, standard deviation.

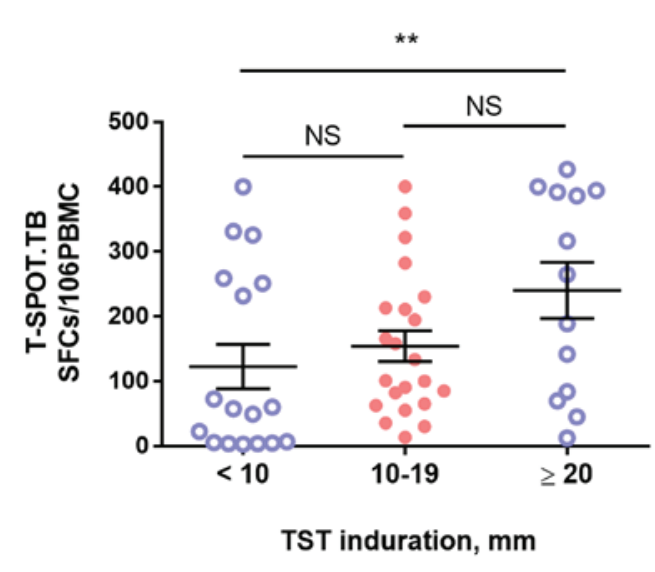

Figure 1. Correlation between the size of the TST induration and T-SPOT.TB result. ${ }^{* *} \mathrm{P}=0.008$; NS, not significant; TST, tuberculin skin test; PBMC, peripheral blood mononuclear cell; SFCs, spot-forming-cells.

$>20 \mathrm{~mm}$ had a higher number of gamma interferon-producing $\mathrm{T}$ cells than those with a negative TST result, which implied that the body had a strong immune response to the exposure to TB bacilli. However, when the TST size was $<20 \mathrm{~mm}$, the correlation between the number of $\mathrm{T}$ cells producing gamma interferon among PBMCs and the size of the TST spot was not high. One possible explanation is that a positive TST test cannot differentiate between $M$. tuberculosis infection, prior BCG vaccination and exposure to NTM (22), particularly when the TST induration is $<15 \mathrm{~mm}(23,24)$. In addition, the TST and the T-SPOT.TB test are designed to detect the presence of $M$. tuberculosis-specific T-cell responses (25) and represent indirect evidence for past or present exposure to TB bacilli. Neither a positive TST size nor T-SPOT.TB result may discriminate active TB infection from LTBI. In light of the high TB burden in China, even if the TST result is strongly positive, a diagnosis of active TB still requires further examination and comprehensive consideration.

There are certain limitations of the present study. First, as a retrospective study, the decision to perform the T-SPOT. TB test and TST depended on the physician's judgment at that time, possibly introducing a selection bias. Second, the number of cases included is limited. On the one hand, as a commercial test, the T-SPOT.TB test has been introduced at our hospital only recently, so the number of cases is not high. On the other hand, the number of patients diagnosed with active TB infection is also not large (pulmonary TB, 15 cases and extrapulmonaryTB, 15 cases). Considering these limitations, the results of the present study should be interpreted with caution. A population-based study with a sufficient sample size and follow-up is required to fully compare the performance of IGRAs and the TST in high-risk TB populations (26).

In conclusion, the T-SPOT.TB test had a higher sensitivity than the TST. An increased TST spot size was associated with a trend toward an increased rate of T-SPOT.TB positivity. However, neither the T-SPOT.TB test nor the TST was sufficiently accurate to be used for detecting active TB disease. Given the comparable performance, the selection of TST or T-SPOT.TB should rather depend on other considerations, including cost, benefits and resources.

\section{Acknowledgements}

The authors wish to thank Professor Chao Cao (Department of Respiratory Medicine, Ningbo First Hospital, Ningbo, China) for his constant support during the course of this work.

\section{Funding}

No funding was received.

\section{Availability of data and materials}

The datasets used or analyzed during the current study are available from the corresponding author on reasonable request.

\section{Authors' contributions}

JY, WK and NX were responsible for the acquisition, analysis and interpretation of the data and contributed to the drafting of the manuscript. XC provided statistical support and data interpretation. $\mathrm{XH}$ and $\mathrm{XC}$ made critical revisions to the manuscript for important intellectual content and performed 
the final proofing. All authors read and approved the final version of the manuscript.

\section{Ethics approval and consent to participate}

The present study was performed with the informed consent of each subject and with the approval of the local Ethics Committee of Ningbo First Hospital (Ningbo, China).

\section{Patient consent for publication}

Not applicable.

\section{Competing interests}

The authors declare that they have no competing interests.

\section{References}

1. Organization WH: Global tuberculosis report 2018. http://www. who.int/tb/publications/global_report/en/. Accessed December 26, 2018.

2. Dinnes J, Deeks J, Kunst H, Gibson A, Cummins E, Waugh N Drobniewski F and Lalvani A: A systematic review of rapid diagnostic tests for the detection of tuberculosis infection. Health Technol Assess 11: 1-196, 2007.

3. Pai M, Zwerling A and Menzies D: Systematic review: T-cell-based assays for the diagnosis of latent tuberculosis infection: An update. Ann Intern Med 149: 177-184, 2008.

4. Sun L, Xiao J, Miao Q, Feng WX, Wu XR, Yin QQ, Jiao WW, Shen C, Liu F, Shen D and Shen AD: Interferon gamma release assay in diagnosis of pediatric tuberculosis: A meta-analysis-gamma. FEMS Immunol Med Microbiol 63: 165-173, 2011.

5. Sester M, Sotgiu G, Lange C, Giehl C, Girardi E, Migliori GB, Bossink A, Dheda K, Diel R, Dominguez J, et al: Interferon- $\gamma$ release assays for the diagnosis of active tuberculosis: A systematic review and meta-analysis- $\gamma$ A. Eur Respir J 37: 100-111, 2011.

6. Lu P, Chen X, Zhu LM and Yang HT: Interferon-gamma release assays for the diagnosis of tuberculosis: A systematic review and meta-analysis. Lung 194: 447-458, 2016.

7. Diagnostic standards and classification of tuberculosis in adults and children. This official statement of the American thoracic society and the centers for disease control and prevention was adopted by the ats board of directors, July 1999. This statement was endorsed by the council of the infectious disease society of America, September 1999. Am J Respir Crit Care Med 161: 1376-1395, 2000.

8. Im JG, Itoh H, Shim YS, Lee JH, Ahn J, Han MC and Noma S: Pulmonary tuberculosis: CT findings-early active disease and sequential change with antituberculous therapy. Radiology 186: 653-660, 1993.

9. Im JG, Webb WR, Han MC and Park JH: Apical opacity associated with pulmonary tuberculosis: High-resolution CT findings. Radiology 178: 727-731, 1991.

10. Liang QL, Shi HZ, Wang K, Qin SM and Qin XJ: Diagnostic accuracy of adenosine deaminase in tuberculous pleurisy: A meta-analysis. Respir Med 102: 744-754, 2008.

11. Pai M, Riley LW and Colford JM Jr: Interferon-gamma assays in the immunodiagnosis of tuberculosis: A systematic review. Lancet Infect Dis 4: 761-776, 2004.
12. Diel R, Loddenkemper R and Nienhaus A: Evidence-based comparison of commercial interferon-gamma release assays for detecting active TB: A metaanalysis. Chest 137: 952-968, 2010.

13. Kang WL, Wang GR, Wu MY, Yang KY, Er-Tai A, Wu SC, Geng SJ, Li ZH, Li MW, Li L and Tang SJ: Interferon-gamma release assay is not appropriate for the diagnosis of active tuberculosis in high-burden tuberculosis settings: A retrospective multicenter investigation. Chin Med J (Engl) 131: 268-275, 2018.

14. Pai M and Menzies D: Interferon-gamma release assays: What is their role in the diagnosis of active tuberculosis? Clin Infect Dis 44: 74-77, 2007.

15. Metcalfe JZ, Everett CK, Steingart KR, Cattamanchi A, Huang L, Hopewell PC and Pai M: Interferon- $\gamma$ release assays for active pulmonary tuberculosis diagnosis in adults in low- and middle-income countries $\gamma$ : Systematic review and meta-analysis. J Infect Dis 204 (Suppl 4): S1120-S1129, 2011.

16. Fan L, Chen Z, Hao XH, Hu ZY and Xiao HP: Interferon-gamma release assays for the diagnosis of extrapulmonary tuberculosis: A systematic review and meta-analysis. FEMS Immunol Med Microbiol 65: 456-466, 2012.

17. Goletti D, Carrara S, Butera O, Amicosante M, Ernst M, Sauzullo I, Vullo V, Cirillo D, Borroni E, Markova R, et al: Accuracy of immunodiagnostic tests for active tuberculosis using single and combined results: A multicenter TBNET-study. PLoS One 3: e3417, 2008.

18. Dosanjh DP, Hinks TS, Innes JA, Deeks JJ, Pasvol G, Hackforth S, Varia H, Millington KA, Gunatheesan R, Guyot-Revol V and Lalvani A: Improved diagnostic evaluation of suspected tuberculosis. Ann Intern Med 148: 325-336, 2008.

19. Cruz AT and Starke JR: Relationship between tuberculin skin test (TST) size and interferon gamma release assay (IGRA) result: When should clinicians obtain IGRAs in children with positive TSTs? Clin Pediatr (Phila) 53: 1196-1199, 2014.

20. Leung CC, Yam WC, Yew WW, Ho PL, Tam CM, Law WS, Wong MY, Leung M and Tsui D: Comparison of T-Spot.TB and tuberculin skin test among silicotic patients. Eur Respir J 31: 266-272, 2008

21. Mahan CS, Johnson DF, Curley C and van der Kuyp F: Concordance of a positive tuberculin skin test and an interferon gamma release assay in bacille Calmette-Guérin vaccinated persons. Int J Tuberc Lung Dis 15: 174-178, i, 2011.

22. Gualano G, Mencarini P, Lauria FN, Palmieri F, Mfinanga S, Mwaba P, Chakaya J, Zumla A and Ippolito G: Tuberculin skin test-Outdated or still useful for Latent TB infection screening? Int J Infect Dis 80S: S20-S22, 2019.

23. Tissot F, Zanetti G, Francioli P, Zellweger JP and Zysset F: Influence of bacille Calmette-Guérin vaccination on size of tuberculin skin test reaction: To what size? Clin Infect Dis 40: 211-217, 2005.

24. Mazurek GH, Lobue PA, Daley CL, Bernardo J, Lardizabal AA, Bishai WR, Iademarco MF and Rothel JS: Comparison of a whole-blood interferon gamma assay with tuberculin skin testing for detecting latent Mycobacterium tuberculosis infection. JAMA 286: 1740-1747, 2001.

25. Mack U, Migliori GB, Sester M, Rieder HL, Ehlers S, Goletti D, Bossink A, Magdorf K, Hölscher C, Kampmann B, et al: LTBI: latent tuberculosis infection or lasting immune responses to M. tuberculosis? A TBNET consensus statement. Eur Respir J 33: 956-973, 2009.

26. Auguste P, Tsertsvadze A, Pink J, Court R, McCarthy N, Sutcliffe $\mathrm{P}$ and Clarke A: Comparing interferon-gamma release assays with tuberculin skin test for identifying latent tuberculosis infection that progresses to active tuberculosis: Systematic review and meta-analysis. BMC Infect Dis 17: 200, 2017. 